\title{
Perfil de uso de metilfenidato e correlatos entre estudantes de medicina
}

\author{
Methylphenidate and correlates use profile among medical students
}

Perfil de uso de metilfenidato y correlación entre estudiantes de medicina

Nathalia Bufaiçal Rassi Carneiro', Daniela Alves dos Santos Gomes ${ }^{1}$, Leonardo Luiz Borges ${ }^{1,2 *}$.

\section{RESUMO}

Objetivo: Observar o perfil de uso de metilfenidato e correlatos (MFC) entre estudantes de medicina e avaliar variáveis relacionadas a qualidade do sono e prática de atividade física. Métodos: Foi realizado um estudo transversal com 180 estudantes de medicina via formulário online em maio de 2020. Para análise dos dados foram empregados os testes do $X^{2}$ e teste exato de Fisher, utilizando o software Past versão 4.03. Resultados: A prevalência do uso de MFC foi de $23,3 \%$, sendo $38,9 \%(n=16)$ do sexo masculino e $61,9 \%$ $(n=26)$ do sexo feminino. Observou-se que $57,1 \%(n=24)$ tiveram diagnóstico médico com equivalente prescrição de algum dos fármacos, enquanto $42,9 \%(n=18)$ fizeram uso não prescrito. Os medicamentos comerciais citados foram: Ritalina ${ }^{\circledR}$, Venvanse ${ }^{\circledR}$, Concerta ${ }^{\circledR}$ e Adderal ${ }^{\circledR}$. Os principais efeitos adversos relatados foram insônia $(62,1 \%)$, taquicardia $(58,6 \%)$, ansiedade $(51,7 \%)$, alteração do apetite $(51,7 \%)$, estresse $(41,4 \%)$, tremores $(41,4 \%)$, boca seca $(34,5 \%)$ e abstinência $(17,2 \%)$. Conclusão: Conclui-se que 0 uso de psicoestimulantes cerebrais sem prescrição médica foi elevado. Devido as reações adversas desses fármacos, existe risco à saúde dos estudantes que fazem seu uso.

Palavras-chave: Metilfenidato, Acadêmicos, Medicina, Automedicação, Insônia.

\begin{abstract}
Objective: To observe the profile of the use of methylphenidate and correlates (CFM) among medical students and to evaluate variables related to sleep quality and physical activity. Methods: A cross-sectional study was carried out with 180 medical students via online form in May 2020. For data analysis, the X2 tests and Fisher's exact test were used, using the software Past version 4.03. Results: The prevalence of MFC use was $23.3 \%$, with $38.9 \%(n=16)$ being male and $61.9 \%(n=26)$ being female. It was observed that $57.1 \%(n=24)$ had a medical diagnosis with equivalent prescription of any of the drugs, while $42.9 \%(n=18)$ used it without prescription. The commercial drugs mentioned were: Ritalina $\AA$, Venvanse $\AA$, Concerta $\AA$ and Adderal $\AA$. The main adverse effects reported were insomnia $(62.1 \%)$, tachycardia $(58.6 \%)$, anxiety $(51.7 \%)$, changes in appetite $(51.7 \%)$, stress $(41.4 \%)$, tremors $(41.4 \%)$, dry mouth $(34.5 \%)$ and abstinence $(17.2 \%)$. Conclusion: It is concluded that the use of brain psychostimulants without medical prescription was high. Due to the adverse reactions of these drugs, there is a risk to the health of the students who use them.
\end{abstract}

Keywords: Methylphenidate, Academics, Medicine, Self-medication, Insomnia.

\section{RESUMEN}

Objetivo: Observar el perfil del uso de metilfenidato y correlatos (CFM) entre estudiantes de medicina y evaluar variables relacionadas con la calidad del sueño y la actividad física. Métodos: se realizó un estudio transversal con 180 estudiantes de medicina vía formulario online en mayo de 2020. Para el análisis de los datos se utilizaron las pruebas X2 y la prueba exacta de Fisher, utilizando el software Past versión 4.03. Resultados: La prevalencia del uso de MFC fue del $23,3 \%$, siendo el $38,9 \%(n=16)$ hombres y el $61,9 \%(n$ $=26)$ mujeres. Se observó que el $57,1 \%(n=24)$ tenía un diagnóstico médico con prescripción equivalente de alguno de los fármacos, mientras que el 42,9\% $(n=18)$ lo usaba sin receta. Los medicamentos comerciales mencionados fueron: Ritalina $\AA$, Venvanse $\AA$, Concerta $\AA$ y Adderal ${ }^{\circledR}$. Los principales efectos adversos reportados fueron insomnio $(62,1 \%)$, taquicardia $(58,6 \%)$, ansiedad $(51,7 \%)$, alteraciones del apetito $(51,7 \%)$, estrés $(41,4 \%)$, temblores $(41,4 \%)$, sequedad de boca $(34,5 \%)$ y abstinencia $(17,2 \%)$. Conclusión: Se concluye que el uso de psicoestimulantes cerebrales sin prescripción médica fue elevado. Debido a las reacciones adversas de estos medicamentos, existe un riesgo para la salud de los estudiantes que los utilizan. Palabras clave: Metilfenidato, Académicos, Medicina, Automedicación, Insomnio.

1 Pontifícia Universidade Católica de Goiás (PUC-Goiás), Goiânia - GO.

*E-mail: leonardo.cbb@pucgoias.edu.br

2 Universidade Estadual de Goiás, Brasil, Anápolis - GO. 


\section{INTRODUÇÃO}

O metilfenidato e outros compostos correlatos constituem as principais substâncias empregadas para o tratamento do Transtorno do Déficit de Atenção e Hiperatividade (TDAH). O metilfenidato representa a substância psicoativa mais utilizada no mundo, entretanto o uso irracional deste fármaco e compostos correlatos consiste em um dos grandes problemas de saúde pública enfrentados pela comunidade médica (ONU, 2011).

Existem evidências que apontam para um crescimento global do consumo de metilfenidato e que este aumento está relacionado principalmente ao seu uso não terapêutico, desvinculado de diagnósticos confirmados. Autoridades sanitárias alegam, ainda, que existem indícios de abuso e de desvio de utilização para outras finalidades, como o aumento da capacidade de concentração, melhora cognitiva, redução da fadiga, dentre outros (ESHER ACT, 2017).

O uso inadequado contribui diretamente para a ocorrência de intoxicações, o que é corroborado pelo Sistema Nacional de Informações Tóxico-Farmacológicas (Sinitox), relata que somente em 2012, esses medicamentos foram responsáveis por $27,27 \%$ dos casos de intoxicação no Brasil. Além disso, o Brasil é um dos países que possui crescente importação de metilfenidato. Segundo dados da ONU de 2015, a importação sofreu um aumento de 300\% de 2012 para 2013 (GOMES RS, et al., 2019). Nessa mesma perspectiva, o Brasil também é o segundo maior consumidor de Ritalina® (cloridrato de metilfenidato) no mundo, perdendo a primeira colocação apenas para os EUA (CARDOSO CA, et al., 2017).

Moynihan R, et al. (2013), aponta que existe preocupação com o excesso de diagnósticos de TDAH, o que pode estar associado a sobrediagnósticos, resultado de problemas relacionados à medicalização excessiva e o tratamento excessivo subsequente, desvirtuamento de diagnóstico, alteração de limiares, mercantilização de doença) e consequente utilização desses medicamentos de maneira inapropriada. No trabalho de Arruda MA (2011), sobre o uso de metilfenidato em crianças e adolescentes para tratamento de TDAH, foi constatado que $60 \%$ dos indivíduos não haviam sequer recebido o diagnóstico correto.

Outro problema associado a esses fármacos, consiste na sua utilização para aumento das funções cognitivas em pessoas saudáveis, especialmente entre estudantes e jovens trabalhadores, devido às suas propriedades psicoestimulantes. Alguns admitem consumi-lo como verdadeiro gatilho para a obtenção de "melhorias" no trabalho e na escola, chamando-os de 'drogas da inteligência'. Em períodos de provas, preparatórios, vésperas de testes, esses indivíduos utilizam essa medicação para aprimorar os seus rendimentos em atividades intelectuais que causam elevada taxa de estresse. (CARVALHO TRF, et al., 2014).

A forma de obtenção deste medicamento pelos alunos, ocorre principalmente de maneira ilícita, por meio de amigos, parentes ou prescrições falsas. Este contexto de automedicação constitui grave problema de saúde pública. Permitir que estudantes de Medicina e médicos tenham livre acesso ao metilfenidato pode comprometer a performance desses indivíduos ao exercerem sua profissão, o que coloca em risco a vida de seus pacientes, além de distorcer a capacidade de discernir com precisão seu nível de competência sem o medicamento (NETO FCCV, et al., 2018).

O objetivo deste estudo foi descrever os principais fatores que podem estar associados ao consumo de metilfenidato e correlatos (MFC) entre estudantes de Medicina de uma Universidade da região central do estado de Goiás.

\section{MÉTODOS}

Este é um estudo quantitativo transversal entre estudantes de Medicina de uma Universidade da região central do estado de Goiás matriculados em 2020. Foram eleitos para participar do estudo todos os alunos do primeiro ao sexto ano, com exceção dos alunos cursando o $8^{\circ}$ e $12^{\circ}$ períodos. Este estudo foi aprovado pelo Comitê de Ética em Pesquisa (CEP) da Pontifícia Universidade Católica de Goiás (Parecer no: 3.710.174; CAAE: 24622619.9.0000.0037).

Para coleta de dados, utilizou-se um questionário gerado pela plataforma Google Forms com modelo padronizado com questões objetivas. Este foi enviado aos alunos por meio de plataformas digitais em maio de 2020, aceitando respostas por até 48 horas desde o envio. 
Os participantes concordaram com o Termo de Consentimento Livre e Esclarecido antes de responder ao questionário e receberam uma via por e-mail em seguida. Foram obtidas 180 respostas, sendo todas elas incluídas nas análises de dados.

O questionário visou obter informações sobre o padrão de uso de metilfenidato e fármacos correlatos (MFC): Ritalina $\AA^{\circledR}$ (metilfenidato), Venvanse ${ }^{\circledR}$ (lisdexamfetamina) e Adderal® (anfetamina). Além disso, o questionário era composto de perguntas gerais objetivas como idade, sexo e período do curso e outras perguntas mais específicas, visando avaliar duas variáveis: qualidade do sono e prática de atividade física.

Os dados foram tabulados e posteriormente os gráficos e a análise estatística descritiva foram feitos utilizando o software Excel. Para a avaliação da associação entre o uso de MFC e as variáveis independentes, foram empregados os testes do $X^{2}$ e teste exato de Fisher, utilizando o software Past versão 4.039 . Foi adotado para os testes estatísticos um nível de significância de $5 \%$.

\section{RESULTADOS}

Foram aplicados 180 questionários por meio de formulários gerados pelo Google Forms. A distribuição de respostas variou conforme período do curso, sendo o $8^{\circ}$ e $12^{\circ}$ períodos excluídos (o $8^{\circ}$ período não possuía turma de alunos no momento da aplicação e o $12^{\circ}$ havia graduado, excepcionalmente, antes do previsto devido às condições de pandemia do COVID-19).

Das respostas, $73,9 \%(n=133)$ foram de estudantes do sexo feminino com idade média de 21,4 (DP=2,45), enquanto o sexo masculino, $26,1 \%(n=47)$, teve uma média de idade de $21 \quad(D P=2,47)$. Em relação a distribuição de respostas por período do curso, houve uma variação de 5 ( $9^{\circ}$ período) a 32 respostas $\left(7^{\circ}\right.$ período) (Tabela 1).

Tabela 1 - Distribuição da amostra de acadêmicos de Medicina de uma Universidade da região central do estado de Goiás

\begin{tabular}{cccc}
\hline Variável & & $\mathbf{N}$ & $\%$ \\
\hline \multirow{2}{*}{ Sexo } & Feminino & 133 & $73,89 \%$ \\
& Masculino & 47 & $26,11 \%$ \\
\hline \multirow{3}{*}{ Período } & 1 & 23 & $12,78 \%$ \\
& 2 & 17 & $9,44 \%$ \\
& 4 & 21 & $11,67 \%$ \\
& 5 & 13 & $7,22 \%$ \\
& 6 & 14 & $7,78 \%$ \\
& 7 & 27 & $15,00 \%$ \\
& 9 & 32 & $17,78 \%$ \\
& 10 & 5 & $2,78 \%$ \\
& 11 & 16 & $8,89 \%$ \\
& 12 & 12 & $6,67 \%$
\end{tabular}

Fonte: Carneiro NBR, et al., 2020.

Quanto aos parâmetros associados à qualidade de vida, dois aspectos principais foram avaliados: os perfis de sono e de prática de atividades físicas dos participantes. A maioria dos estudantes, $58,9 \% \quad(n=106)$, apresentaram, em média, 6-7 horas de sono por noite e 22,78\% ( $n=41)$, em média, 4-5 horas.

Qualitativamente, um total de $47,22 \%$ (n=85) classificou seu sono como "regular", ou seja, em um nível intermediário, variando em uma escala de péssimo a ótimo. Da amostra total, $8,33 \%(n=15)$ referiram fazer uso de medicamentos para dormir, dentre eles melatonina $(n=5)$, quetiapina $(n=5)$, rivotril $(n=3)$ e fitoterápicos $(n=2)$ (Tabela 2). 
Tabela 2 - Aspectos relacionados ao sono da amostra de acadêmicos de Medicina de uma Universidade da região central do estado de Goiás.

\begin{tabular}{cccc}
\hline Variável & & N & $\%$ \\
\hline \multirow{2}{*}{ Horas de sono } & $4-5 \mathrm{~h}$ & 41 & $22,78 \%$ \\
& $6-7 \mathrm{~h}$ & 106 & $58,89 \%$ \\
& $7-8 \mathrm{~h}$ & 31 & $17,22 \%$ \\
& Mais de $8 \mathrm{~h}$ & 2 & $1,11 \%$ \\
\hline \multirow{2}{*}{ Percepção da Qualidade de sono } & Péssima & 10 & $5,56 \%$ \\
& Ruim & 25 & $13,89 \%$ \\
& Regular & 85 & $47,22 \%$ \\
& Boa & 49 & $27,22 \%$ \\
\hline \multirow{2}{*}{ Uso de remédio para dormir } & Ótima & 11 & $6,11 \%$ \\
& Sim & 15 & $8,33 \%$ \\
& Não & 165 & $91,67 \%$ \\
\hline \multirow{2}{*}{ Qual medicamento faz uso } & Melatonina & 5 & $33,33 \%$ \\
& Quetiapiana & 5 & $33,33 \%$ \\
& Rivotril & 3 & $20 \%$ \\
& Fitoterápicos & 2 & $13,33 \%$ \\
\hline
\end{tabular}

Fonte: Carneiro NBR, et al., 2020.

Aproximadamente $30 \%(n=53)$ dos alunos relataram não praticar atividade física e, daqueles que assumiram praticar regularmente, um total de $32,28 \%(n=41)$ praticam exercício físico 1 a 2 vezes por semana, $44,88 \%(n=57), 3$ a 4 vezes por semana, 19,69\% ( $n=25), 5$ a 6 vezes por semana e $4 \%(n=3,15 \%)$, todos os dias (Tabela 3).

Tabela 3 - Perfil de prática de atividade física da amostra de acadêmicos de Medicina de uma Universidade da região central do estado de Goiás.

\begin{tabular}{cccc}
\hline Variável & & $\mathbf{N}$ & $\%$ \\
\hline Prática de atividade física & Sim & 127 & $70,56 \%$ \\
& Não & 53 & $29,44 \%$ \\
\hline Frequência na prática de atividade física & $1-2 x / s e m$ & 41 & $32,28 \%$ \\
& $3-4 x / s e m$ & 57 & $44,88 \%$ \\
& $5-6 x /$ sem & 25 & $19,69 \%$ \\
& Todos os dias & 4 & $3,15 \%$
\end{tabular}

Fonte: Carneiro NBR, et al., 2020.

A prevalência do uso de metilfenidato e correlatos em algum momento da vida foi de $23,3 \%$ por estudantes da amostra, sendo $34,04 \%(n=16)$ e $19,55 \%(n=26)$ no sexo masculino e feminino, respectivamente. Os medicamentos comerciais citados foram: Ritalina $\AA$, Venvanse $\AA$, Concerta $\AA$ e Adderal $\AA$, sendo os dois primeiros os mais utilizados, representando $73,81 \%(n=31)$ e $42,86 \%(n=18)$, respectivamente. Quanto a frequência de uso, cerca de metade dos estudantes $(n=22)$ referiram fazer uso aleatório em festas, raves e preparatórios para provas e $35,7 \%(n=15)$ fazem uso regular de 5 a 7 vezes por semana (Tabela 4$)$. 
Tabela 4 - Perfil de uso de metilfenidato e correlatos da amostra de acadêmicos de Medicina de uma Universidade da região central do estado de Goiás.

\begin{tabular}{|c|c|c|c|}
\hline Variável & & $\mathbf{N}$ & $\%$ \\
\hline \multirow{2}{*}{ Já fez uso de MFC } & Sim & 42 & $23,33 \%$ \\
\hline & Não & 138 & $76,67 \%$ \\
\hline \multirow{4}{*}{ Quais anfetaminas já fez uso } & Ritalina $\AA$ & 31 & $73,81 \%$ \\
\hline & ${ }^{*}$ Concerta $®$ & 10 & $23,81 \%$ \\
\hline & Venvance ${ }^{\circledR}$ & 18 & $42,86 \%$ \\
\hline & Adderal® & 1 & $2,38 \%$ \\
\hline \multirow{3}{*}{ Qual a frequência de uso de AF? } & $3-4 x /$ sem & 5 & $11,90 \%$ \\
\hline & $5-7 x /$ sem & 15 & $35,71 \%$ \\
\hline & Uso aleatório & 22 & $52,38 \%$ \\
\hline
\end{tabular}

Legenda: Um participante pode ter feito o uso de mais de uma substância.

Fonte: CARNEIRO NBR, et al., 2020.

Em relação ao uso prescrito e não prescrito desses medicamentos, 57,1\% ( $n=24)$ alegaram apresentar diagnóstico médico com equivalente prescrição de algum fármaco desta categoria, enquanto os demais fazem uso não prescrito. Da parcela sob uso prescrito, os diagnósticos médicos apontados foram transtorno de déficit de atenção $(79,2 \%, n=19)$, hiperatividade $(12,5 \%, n=3)$ e narcolepsia $(8,3 \%, n=2)$.

O principal motivo alegado para o consumo de psicoestimulantes foi o aumento da capacidade de concentração $(64,29 \%, n=27)$. Além desse, os estudantes também assinalaram condição de saúde controlada e comprovada ( $33,3 \%, n=14)$, redução do sono/fadiga $(23,8 \%, n=10)$, aumento da sensação de prazer $(11,9 \%$, $n=5)$ e perda de peso $(2,4 \%, n=1)$ como motivação para o uso de metilfenidato e derivados.

Quanto aos efeitos adversos observados pelos estudantes após o uso desses medicamentos ( $n=29)$, os principais sintomas foram insônia $(62,1 \%, n=18)$, taquicardia $(58,6 \%, n=17)$, ansiedade $(51,7 \%, n=15)$, alteração do apetite $(51,7 \%, n=15)$, estresse $(41,4 \%, n=12)$, tremores $(41,4 \%, n=12)$, boca seca $(34,5 \%, n=10)$ e abstinência $(17,2 \%, n=5)$.

Dois participantes assinalaram também a alternativa 'outros', alegando alterações intestinais negativas $(3,4 \%, n=1)$ como sintoma após o uso. Apenas 1 estudante relatou não ter apresentado nenhum efeito adverso $(3,4 \%, n=1)$. Dos estudantes que já haviam feito uso de alguma anfetamina na vida $(n=42), 66,7 \%$ afirmaram que já usavam antes de ingressar na faculdade. Os 33,3\% restantes, porém, tiveram o primeiro contato com a droga após entrar na faculdade.

A amostra total, isto é, os 180 participantes da pesquisa foram, ainda, questionados a respeito do conhecimento sobre o mecanismo de ação das anfetaminas. Desses, 42,2\% ( $n=76)$ desconhecem o efeito dessas substâncias no organismo.

De acordo com a distribuição por ano do curso dos alunos que disseram já ter feito uso de MFC, constatouse que o terceiro ano ( $5^{\circ}$ e $6^{\circ}$ períodos) tem a maior porcentagem, com uma taxa de $28,57 \%$, seguido pelo primeiro ano ( $1^{\circ}$ e $2^{\circ}$ períodos), com $23,8 \%$.

Após a aplicação do teste do qui-quadrado para verificação da associação entre as variáveis sexo e frequência de uso de metilfenidato e correlatos, o sexo masculino apresentou maior quantidade de indivíduos $(34,04 \% ; n=16)$ que declararam ter utilizado algum dos medicamentos investigados em relação às mulheres $(19,55 \%, n=26)$ do sexo feminino. Existe uma associação significativa ao nível de $5 \%$ entre a variável sexo e frequência de uso dos medicamentos supracitados $(p=0,04)$ (Figura 1A). 
Figura 1 - Resultados do perfil de uso de metilfenidato e correlatos em estudantes de Medicina de uma Universidade da região central do estado de Goiás.
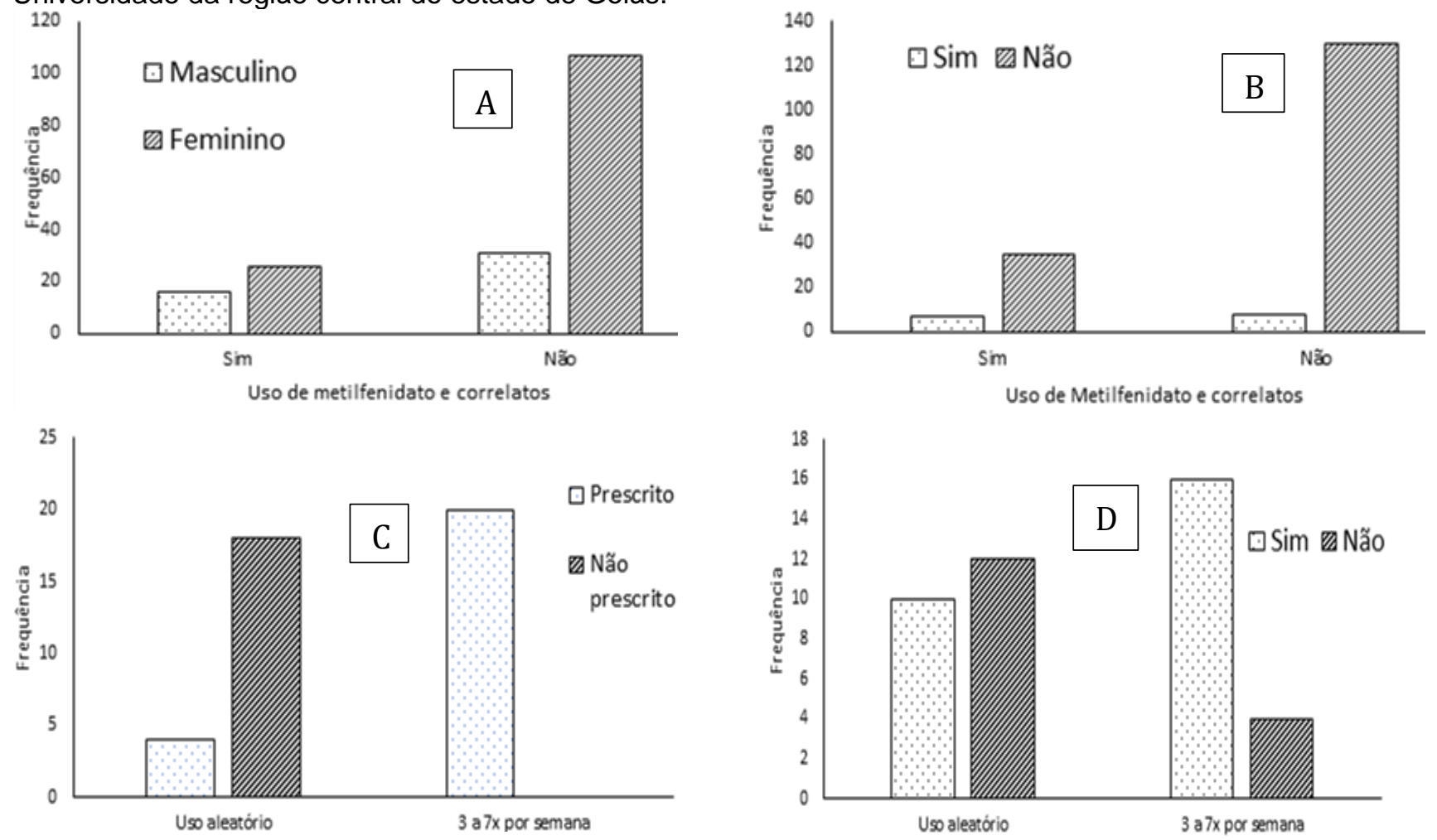

Legenda: A-Distribuição de alunos que já fizeram uso de metilfenidato e correlatos e que não fizeram uso de acordo com o sexo $(p=0,04)$. B-Distribuição de alunos que já fizeram uso de metilfenidato e correlatos e não fizeram em relação ao uso de medicação para dormir ou não $(p=0,02)$. C- Distribuição de alunos em uso aleatório e em uso regular ( 3 a 7 vezes por semana) de metilfenidato e correlatos em relação ao uso prescrito e não prescrito dessas drogas $(p<0,05)$. D- Distribuição de alunos em uso aleatório e em uso regular ( 3 a 7 vezes por semana) de metilfenidato e correlatos em relação ao conhecimento e desconhecimento do mecanismo de ação dessas drogas $(p=0,02)$.

Fonte: Carneiro NBR, et al., 2020.

O período do curso, estatisticamente, não gerou diferenças significativas com relação ao uso ou não de metilfenidato e correlatos, ao total de horas dormidas ou a qualidade do sono dos estudantes. Observou-se, também, que o fator idade não teve influência significativamente importante sobre o uso ou não desses medicamentos.

Dentre os 15 participantes que relataram utilizar algum medicamento para dormir, 7 acadêmicos (46,6\%) também fazem ou já fizeram uso de metilfenidato e correlatos. Entretanto, dos 165 participantes que relataram não utilizar medicamentos hipnóticos, apenas 35 (21,21\%) alegam fazer ou já ter feito uso de metilfenidato e correlatos alguma vez na vida. Dessa forma, observa-se uma maior prevalência de uso de medicamentos hipnóticos entre os indivíduos que relataram utilizar metilfenidato e correlatos e esta associação mostrou-se significativa $(p=0,02)$ (Figura 1B).

Não foi encontrada associação significativa ao nível de $5 \%$ entre as frequências de efeitos adversos com o fato do metilfenidato e correlatos serem obtidos ou não por meio de prescrição médica. Portanto, independente do estudante ter obtido esses medicamentos por meio de prescrição médica ou não, a probabilidade de ocorrência de efeitos adversos não é significativamente diferente $(p>0,05)$

Foi avaliada a relação entre o uso prescrito e não prescrito de metilfenidato e correlatos e a frequência de uso de tais medicações. Por meio do teste exato de Fisher, observou-se relação estatisticamente significativa ao nível de $1 \%$, ou seja, estudantes sob uso prescrito tem geralmente uma frequência de uso maior desses medicamentos (3 a 7 vezes por semana) (Figura 1C). 
Entre aqueles estudantes que fazem uso frequente desses medicamentos de 3 a 7 vezes por semana $(n=20)$, a maioria referiu conhecer o mecanismo dessas drogas $(80 \%, n=16)$. Enquanto do grupo em uso aleatório ( $n=22)$, apenas $45 \%$ referiu ter esse conhecimento. Observou-se, portanto, maior conhecimento do mecanismo de ação do metilfenidato e correlatos destes em relação àqueles $(p=0,02)$ (Figura 1D).

\section{DISCUSSÃO}

A prevalência de uso encontrada nesta pesquisa $(23,33 \%)$ apresentou concordância com uma prevalência de $20 \%$ observada por Morgan $\mathrm{HL}$, et al. (2017), em alunos de uma faculdade pública de Medicina do extremo sul do Brasil. Entretanto, a porcentagem obtida neste estudo foi inferior ao encontrado por Menezes ASS, et al., (2017), que apontaram um total de 54\% em estudantes de outras áreas da saúde (Enfermagem, Farmácia e Odontologia) de Rondônia.

Teter CJ, et al. (2006), conduziram um estudo em universidades do sudoeste dos EUA e encontraram uma porcentagem de $9 \%$ de acadêmicos que declararam utilizar metilfenidato e fármacos correlatos. Percebe-se, dessa forma, que existe uma variabilidade ( $9 \%$ a $54 \%$ ) no comportamento de cada amostra estudada, de acordo com a região investigada. Silveira VI, et al. (2015), observou resultados semelhantes em um estudo realizado em uma universidade privada no sul de Minas Gerais em relação ao padrão de frequência de uso de acordo com cada período da faculdade (apesar de algumas variações), uma tendência decrescente do consumo de metilfenidato ao longo do curso.

Uma hipótese para esse comportamento seria a brusca mudança de rotina ao ingressar na faculdade e o ambiente altamente competitivo e rígido que a Medicina impõe, além da alta carga horária do curso (mínimo 7.200 horas, segundo o Ministério da Educação, sendo o curso com maior carga horária no Brasil), que exerce uma desgastante rotina aos estudantes, podendo levá-los a recorrer ao uso de substâncias que possam aumentar sua capacidade (BRASIL, 2004).

$\mathrm{Na}$ mesma perspectiva, pode-se justificar o decréscimo do uso nos períodos finais pela adaptação às exigências que se cria ao longo da vida universitária. No entanto, alguns autores também encontraram tendências crescentes nos anos finais do curso de Medicina, como Morgan HL, et al. (2017), principalmente em virtude da maior busca pela qualificação profissional e inscrição em provas para ingresso em residências médicas, resultando no maior índice do consumo de psicoestimulantes.

Este estudo sugere também, existir uma tendência maior significativa $(p<0,05)$ de usuários do sexo masculino dentre os graduandos de Medicina da Universidade investigada $(34,04 \%)$ quando comparado ao sexo feminino (19,55\%), em concordância com os resultados obtidos em estudantes de medicina da Universidade Federal da Bahia ( $p=0,01$ ) (Cruz TCSC, et al. (2011). Por outro lado, Silveira VI, et al. (2015) e Carneiro SG, et al. (2013) não encontraram diferença significativa entre os sexos. Ademais, uma revisão de literatura de Lage DC, et al. (2015), composta por 18 artigos revelou que não há diferenças significativas de uso não prescrito entre os gêneros.

As motivações para o consumo de MFC foram similares ao encontrado por estudos anteriores (CRUZ TCSC, et al., 2011; MENEZES ASS, et al., 2017; MORGAN HL, et al., 2017; SILVEIRA VI, et al., 2015). Mais de $60 \%$ da amostra $(64,29 \%)$ que já fez uso dessas substâncias alega o aumento da capacidade de concentração como motivador principal, semelhante ao reportado por Cruz TCSC, et al. (2011), o que reforça a necessidade de os estudantes aumentarem suas capacidades produtivas no ambiente de alta pressão e competitividade dentro do curso de Medicina.

De fato, o "aperfeiçoamento cognitivo" é visado pela maioria dos estudantes, mesmo saudáveis, como estímulo para o uso de psicoestimulantes. Esses dados subsidiam a hipótese de que o curso de Medicina, pode ser visto como um fator de risco para o uso de substâncias psicoativas, concordando com Posada J, (2016).

É provável que a necessidade de longos períodos de estudo, o cansaço físico, o estresse e a competitividade contribuam para que este curso de graduação seja considerado de risco dentro deste contexto. A avaliação da qualidade do sono entre acadêmicos de Medicina é uma preocupação importante devido ao impacto no estado emocional e no nível de produtividade dos estudantes (BENAVENTE SBT, et al., 2014). Corrêa CC, et al. (2017), demostraram que acadêmicos de Medicina estão mais sujeitos a distúrbios do sono, especialmente estudantes dos primeiros períodos. 
Neste mesmo estudo, foi revelada uma tendência semelhante aos dados aqui apresentados, especialmente no tempo em horas de sono que os estudantes relataram, em que $68 \%$ declararam apresentar de 6 a 7 horas de sono, enquanto que os acadêmicos de Medicina da Universidade investigada declararam em sua maioria $(58,9 \%)$ apresentar este mesmo padrão de sono. Da mesma forma, a prática de atividade física interfere diretamente no bem-estar geral dos estudantes. Em concordância com Peleias MDA, (2018) que observou que $39,5 \%$ dos estudantes de Medicina não realizavam qualquer tipo de atividade física em tempo de lazer, 29,44\% dos estudantes deste estudo relataram não praticar qualquer exercício21.

Ambas as prevalências, no entanto, são superiores a encontrada por Dumith SC, et al. (2011) que analisou 300.000 indivíduos em 76 países, sugerindo que a prevalência mundial de inatividade física seja de 21,4\%. Esta comparação suscita uma preocupação quanto à saúde destes estudantes inseridos em um contexto de pouco incentivo ao cuidado com a própria saúde.

Um dado preocupante obtido nesta pesquisa foi a prevalência de estudantes que alegaram fazer uso não prescrito (off-label) de metilfenidato e correlatos (42,85\%), cerca de $12 \%$ a mais do que o apontado por Bogle $\mathrm{KE}$, et al. (2009). A prescrição desse medicamento, de acordo com a Portaria $n^{\circ} 344 / 199824$ que regulamenta as substâncias de controle especial, exige notificação de receita $\mathrm{A}$ (cor amarela), com o intuito de controlar e restringir o uso apenas para casos necessários. Este resultado, portanto, vai ao encontro do panorama encontrado por Aquino AA, et al. (2015) na Universidade Federal de Pelotas, em que os meios mais frequentes de obtenção de remédios controlados de maneira ilegal foram: através amigos e/ou familiares sem receita médica $(42,4 \%)$ e com receita médica comprada ou recebida sem consulta prévia ao especialista $(12,1 \%)$.

Nessa mesma perspectiva, contrariando um estudo norte-americano feito por Teter CJ, et al. (2003), com 3500 estudantes em que apenas $21 \%$ da amostra havia começado a fazer uso de MFC antes mesmo de ingressar na faculdade, esta pesquisa apontou um total de $66,67 \%$ de estudantes na mesma categoria. Isso sustenta a ideia de que os alunos têm o primeiro contato com essa droga já no ensino fundamental/médio, durante o preparatório para provas de vestibular, Santana C (2019) revelou que nesse momento estressante os alunos estão sujeitos a carga horária de estudos extenuantes (média de 3,83 horas extra curso diárias), assim como a cobrança de resultados constantes, o que pode precipitar a busca por essas substancias (BRANT LC, et al., 2012).

Daqueles que alegaram já ter feito uso de MFC alguma vez na vida $(n=42), 26(61,9 \%)$ afirmaram conhecer o mecanismo de ação dessas substâncias. Desses 26 estudantes, 20 responderam ter algum tipo de diagnóstico médico comprovado que justificasse a prescrição desse medicamento. Ao mesmo tempo que esse dado indica certo nível de orientação dos alunos, expõe um possível sobre diagnóstico de TDAH por parte dos médicos, uma vez que cerca de $80 \%$ dos que fazem uso prescrito alegaram ter esse transtorno comprovado.

A suspeita diagnóstica, atualmente, baseia-se em avaliações médicas e psicológicas abrangentes, e o diagnóstico é clínico apoiado, porém apoiado em manuais diagnósticos. Os mais utilizados internacionalmente são a Classificação Internacional de Doenças (CID-10) (OMS, 2008) e o Manual de Diagnóstico e Estatístico dos Transtornos Mentais (DSM-V). A tríade sintomatológica clássica, por sua vez, caracteriza-se por desatenção, hiperatividade e impulsividade. Enquanto a eficácia dos psicoestimulantes no início do tratamento é inegável, tal propriedade tem sido questionada na terapia de longo prazo.

Uma explicação pode ser o desenvolvimento de tolerância, principalmente naqueles que fazem uso de altas doses. Por outro lado, sobre os potenciais benefícios deste medicamento em indivíduos que não sofrem de TDAH, estudos existentes não mostram evidências consistentes de otimização sobre o SNC (BRANT LC, et al.,2012).

Os efeitos colaterais relatados pelos participantes desta pesquisa foram semelhantes ao observado por Menezes ASS, et al. (2017), em Rondônia. A insônia foi o sintoma com maior prevalência em ambos os estudos, com $62,1 \%$ e $38 \%$ dos estudantes alegando a apresentação desse quadro. Affonso RS, et al. (2016), em um estudo com estudantes da área da Saúde da Faculdade Anhanguera de Brasília (FAB), também encontrou resultado similar, com maior relevância para cefaleia (22\%), taquicardia (22\%), insônia (22\%) e boca seca (16\%). 
Sabe-se que o metilfenidato faz parte de um grupo de drogas que atua bloqueando a recaptação de dopamina e de noradrenalina, o que aumenta consideravelmente a densidade desses neurotransmissores nas sinapses. Apesar dos vastos estudos existentes sobre essa droga, ainda existe uma carência de investigação sobre seus efeitos colaterais no organismo, principalmente no que se refere a indivíduos saudáveis fazendo uso off-label, o que pode, inclusive, potencializar a gravidade e a toxicidade desse medicamento.

Os dois fatores relatados anteriormente (efeito ansiogênico de MFC e da pressão exercida progressivamente pelo curso de Medicina) poderiam estar associados e por conseguinte potencializar quadros de ansiedade entre os estudantes (AFFONSO RS, et al., 2016; MENEZES ASS, et al. 2017). Este estudo foi realizado com metodologia compatível com outras pesquisas existentes sobre o tema, em ambientes semelhantes, o que facilitou a comparação de resultados. No entanto, os resultados aqui obtidos representam um comportamento local, que pode ser influenciado também por aspectos culturais e socioeconômicos.

Esta pesquisa, ainda, apresenta dados que são preocupantes quanto ao uso inadequado de MFC e suscita a necessidade de medidas que visem a redução deste problema que afeta decisivamente a saúde e qualidade de vida de estudantes do curso de Medicina, possivelmente ainda trazendo consequências no profissional que será formado. Outros estudos são necessários para aumentar a nossa compreensão evidenciar os possíveis efeitos desses fármacos no organismo, a fim de reduzir as consequências do uso indiscriminado. Todavia, já está claro que o uso não prescrito do metilfenidato por estudantes é uma realidade em nosso país e não pode ser ignorado.

\section{CONSIDERAÇÕES FINAIS}

O uso de psicoestimulantes cerebrais sem prescrição médica entre estudantes de medicina foi elevado. Este resultado gera preocupação devido às diversas reações adversas que os medicamentos desta categoria podem desencadear, representando assim um problema de saúde pública.

\section{REFERÊNCIAS}

1. AFFONSO RS, et al. O uso indiscriminado do cloridrato de metilfenidato como estimulantes por estudantes da área da saúde da Faculdade Anhanguera de Brasília (FAB). Infarma - Ciências Farmacêuticas, 2016; 28(3):166-172.

2. ARRUDA MA. TDAH no Brasil, o que a Folha de SP não mostrou. Instituto Glia, 2011.

3. AQUINO AA, et al. O uso ilegal de psicoestimulantes farmacológicos por estudantes universitários: uma análise a partir dos silenciamentos da série Narcos (2015) e da lei 11.343 DE 2006. XXVIII Congresso Iniciação Científica UFPel, 2019.

4. BENAVENTE SBT, SILVA RM, HIGASHI AB, GUIDO LA, COSTA ALS. Influence of stress factors and sociodemographic characteristics on the sleep quality of nursing students. Revista da Escola de Enfermagem da USP, 2014; 48(3): 514-520.

5. BOGLE KE, SMITH BH. Illicit methylphenidate use: a review of prevalence, availability, pharmacology, and consequences. Current Drug Abuse Reviews, 2009; 2:157-176.

6. BRANT LC, CARVALHO TRF. Metilfenidato: medicamento gadget da contemporaneidade. Interface - Comunicação, Saúde, Educação, 2012; 16(42): 623-636.

7. BRASIL. Ministério da Educação. Carga horária mínima dos cursos de graduação, bacharelados, na modalidade presencial. Brasília: Ministério da Educação, 2004.

8. BRASIL, Ministério da Saúde. Regulamento técnico sobre substâncias e medicamentos sujeitos a controle especial Portaria n. 344. Brasília: Ministério da Saúde,1998.

9. CARDOSO CA, SOUZA NB. O uso irracional da ritalina. Faculdade Atenas, 2017; 1-12.

10. CARNEIRO SG, et al. O uso não prescrito de metilfenidato entre acadêmicos de Medicina. Cadernos UniFOA, 2013; 8(1): 53-59.

11. CARVALHO TRF, et al. Exigências de produtividade na escola e no trabalho e o consumo de metilfenidato. Educação e Sociedade, 2014; 35(127): 587-604.

12. CORRÊA CC, et al. Qualidade de sono em estudantes de medicina: Comparação das diferentes fases do curso. Jornal Brasileiro de Pneumologia, 2017; 43(4): 285-289.

13. CRUZ TCSC, et al. Uso não uso não-prescrito de metilfenidato entre estudantes de medicina da Universidade Federal da Bahia. Gazeta Médica da Bahia, 2011; 81(1): 3-6.

14. DUMITH SC, et al. Worldwide prevalence of physical inactivity and its association with human development index in 76 countries. Preventive Medicine, 2011; 53(1-2): 24-28.

15. ESHER ACT. Rational use of medicines, pharmaceuticalization and uses of methylphenidate. Ciência e Saúde Coletiva. 2017; 22(8): 2571-2580. 
16. GOMES RS, et al. Vendas de metilfenidato: uma análise empírica no Brasil no período de 2007 a 2014. 64a Reunião da Região Brasileira da Sociedade Internacional de Biometria $18^{\circ}$ Simpósio Estatística Aplicada à Experimentação Agronômica (SEAGRO), 2019; 1: 663-681.

17. HAMMER O, HARPER DA. Paleontological Data Analysis. Blackwell, 2005.

18. LAGE DC, et al. Uso de metilfenidato pela população acadêmica: revisão de literatura. Brazilian Journal of Surgery and Clinical Research, 2015; 10(3): 31-39.

19. ORGANIZAÇÃO DAS NAÇÕES UNIDAS - ONU. Report of the International Narcotics Control Board for 2011. State 2010, 2011.

20. MENEZES ASS, et al. O uso de psicoestimulantes por acadêmicos de uma instituição de ensino superior do estado de Rondônia. Repositório Institucional Facimed, 2017; 1-13.

21. MORGAN HL, et al. Consumo de Estimulantes Cerebrais por Estudantes de Medicina de uma Universidade do Extremo Sul do Brasil: Prevalência, Motivação e Efeitos Percebidos. Revista Brasileira de Educação Médica, 2017; 41(1): 102-9.

22. MOYNIHAN R, et al. Winding back the harms of too much medicine. BMJ, 2013; 346: 1-2.

23. PELEIAS MDA. A atividade física e a qualidade vida do estudante de medicina no Brasil. São Paulo, São Paulo. 2018. Doutorado [Tese]. Universidade de São Paulo.

24. POSADA J. Uso y abuso del metilfenidato en Colombia. Ministerio de Salud, Bogota, 1996.

25. SANTANA C. Saúde mental: depressão, ansiedade e estresse de estudantes de pré-vestibular para o curso de Medicina, RS. Trabalho de conclusão de curso (Medicina). Universidade Federal da Fronteira Sul, Passo Fundo, 2019; 45 p.

26. SILVEIRA VI, et al. Uso de psicoestimulantes por acadêmicos de Medicina de uma universidade do sul de Minas Gerais. Revista da Universidade Vale do Rio Verde, 2015;13(2): 186-192.

27. TETER CJ, et al. Illicit use os specific prescription stimulants among college students: prevalence, motives, and routes of administration. Pharmacotherapy, 2006; 26: 1501-1510.

28. TETER CJ, et al. Illicit methylphenidate use in an undergraduate student sample: prevalence and risk factors. Pharmacotherapy, 2003; 23(5): 609-617.

29. VASCONCELOS NETO FCCV, et al. O Uso Não Prescrito De Metilfenidato Entre Acadêmicos De Medicina: Uma Revisão De Literatura. Revista Interdisciplinar em Saúde, Cajazeiras, 2018; 5(4): 759-773. 